\title{
In this number
}

\section{A call for glossaries in public health}

We have decided to start a new section in $\mathcal{F E C H}$. Our culture as a scientific discipline is too much focused on discussing the findings in empirical research following the strong influence on epidemiology of the positivist approach to science. Rarely do you hear or read in our meetings or journals of discussions about how to refine concepts or define basic ideas. Obsessed as we are by results and action, we often forget to build a strong conceptual basis for our endeavours. There is nothing more practical than a good theory, but we lack a robust theoretical base for public health. We use everyday words that evoke different meanings in different minds and nobody seems too worried about it. Just as an example, let us use our identity - public health. Do we all agree on what health means? And, what about the meaning of public?

Words are the way we build up our arguments, defend our thoughts and seek for change. As a consequence, we have developed a specific terminology aimed at clarifying concepts and deepening our understanding of everything that surround us. However, our own jargon often results in the opposite-like a Tower of Babel in which words have become a confusing tool instead of a helping one.

Several years ago, in our pages, Nijhuis and van der Maesen identified at least four different meanings for public health based on the ideological assumptions on the meaning of health and society. ${ }^{1}$ Clearly there is room for conceptualisation and debate, so let us also use $\mathcal{F E C H}$ for this.

Many of our papers make use of very sophisticated, specialised jargon and are extremely difficult to understand for junior researchers and practitioners. Glossaries could be excellent gates to help them enter and develop an interest in new fields.
We are therefore starting a new section in $\mathcal{F E C H}$ dedicated to publishing glossaries in public health. This initiative is aimed both to cover the needs of our non-specialist readers and to contribute to the wider need in our discipline on conceptual debates.

This is a call for papers from any person or group interested in taking part in this new and challenging project. Glossaries to be submitted should contain basic definitions (between 10 and 20) of crucial terms in the field mentioned. Longer glossaries would require to be published in various parts. Definitions should be as clear as possible and understandable for non-initiated audiences. Each definition should be no longer than 50 words. References-if needed-should follow the usual $\mathcal{F E C H}$ format. First authors are encouraged to invite other colleagues to participate as coauthors. The submitted glossaries will follow the usual pathways in $\mathcal{F E C H}$ management of papers, including peer review, but will be published quickly once they have been accepted, in so far as we want each issue to include a glossary.

If you are interested in this initiative, please let us know as soon as possible together with any query you may have.

IRMA MUNOZ-BAELL Department of Linguistics and Literature, University of Alicante

CARLOS ALVAREZ-DARDET Editor

1 Nijhuis HG, van der Maesen LJ. The philosophical foundations of public health: an invitation to debate. $\mathcal{F}$ Epidemiol Community Health 1994;48:1-3. 